\title{
The Role of Forced and Voluntary Training on Accumulation of Neural Cell Adhesion Molecule and Polysialic Acid in Muscle of Mice with Experimental Autoimmune Encephalomyelitis
}

\author{
Farzaneh Torabimehr, ${ }^{1}$ Mohammad Reza Kordi (D), ${ }^{2}$ Reza Nouri, ${ }^{2}$ Jafar Ai, ${ }^{3}$ \\ and Sadegh Shirian ${ }^{4,5,6,7}$ \\ ${ }^{1}$ Department of Neuromuscular Exercise Physiology, Kish International Campus, Tehran University, Kish Island, Iran \\ ${ }^{2}$ Department of Exercise Physiology, University of Tehran, Tehran, Iran \\ ${ }^{3}$ Department of Tissue Engineering and Applied Cell Sciences, School of Advanced Technologies in Medicine, \\ Tehran University of Medical Sciences, Tehran, Iran \\ ${ }^{4}$ Department of Pathology, School of Veterinary Medicine, Shahrekord University, Shahrekord, Iran \\ ${ }^{5}$ Shiraz Molecular Pathology Research Center, Dr Daneshbod Pathol Lab, Shiraz, Iran \\ ${ }^{6}$ Biotechnology Research Institute, Shahrekord University, Shahrekord, Iran \\ ${ }^{7}$ Shefa Khatam Neuroscience Research Center, Tehran, Iran
}

Correspondence should be addressed to Mohammad Reza Kordi; mrkordi@ut.ac.ir

Received 28 August 2019; Revised 26 February 2020; Accepted 4 March 2020; Published 8 April 2020

Academic Editor: Juntra Karbwang

Copyright (C) 2020 Farzaneh Torabimehr et al. This is an open access article distributed under the Creative Commons Attribution License, which permits unrestricted use, distribution, and reproduction in any medium, provided the original work is properly cited.

\begin{abstract}
It has been suggested that depletion of adhesion molecules is one of the factors associated with or possibly responsible for multiple sclerosis (MS) progression. The aim of this study was to investigate the effect of forced and voluntary training before and after induction of experimental autoimmune encephalomyelitis (EAE) on accumulation of neural cell adhesion molecule (NCAM) and polysialic acid (PSA) in neuromuscular junction denervation in plantaris and soleus muscles in C57BL/6 female mice. A total of 40 female C57BL/6 mice, 10-week-old, were randomly divided into four groups, including induced control groups without EAE induction, induced EAE without training, and forced and voluntary training groups. Myelin oligodendrocyte glycoprotein peptide 35-55 (300 $\mu \mathrm{g}$ in saline; MOG 35-55; KJ Ross-Petersen ApS, Denmark) was injected subcutaneously at the base of the tail of each mouse. Clinical assessment of EAE was performed daily using a 15-point scoring system following immunization. Training groups performed the swimming program for $30 \mathrm{~min} /$ day, 5 times/week, for 4 weeks. Mice had access to a treadmill for one hour per day, 5times/week, for 4 weeks in individual cage. The mice were scarified, and the plantaris and soleus muscles were then isolated for investigation of proteins expression using IHC. An analysis of the preventive exercise (before) and recovery exercise (after) of the EAE was performed. Images of the stained sections were taken using a fluorescent microscope. Quantitative image analysis was performed using ImageJ software package. The obtained data from the mean percentage expression of PSA and NCAM in pre- and post-soleus and plantaris muscles showed that the highest and lowest expression levels of PSA and NCAM belonged to control and swim EAE (SE) groups, respectively. The low expression levels of PSA and NCAM were detected in rat with MS without intervention. In conclusion, the relationship between increasing levels of NCAM and PSA protein expression and voluntary and compulsory activity were detectable both in pre and post-soleus and plantaris. However, voluntary activity resulted in more expression levels of NCAM and PSA than that of compulsory. In conclusion, since it has been suggested that depletion of NCAM is one of the factors associated with or possibly responsible for MS progression, these findings show exercise MS progression may be reduced by increasing expression of exercise-related adhesion molecule such as NCAM and PSA (a glycan modification of the NCAM).
\end{abstract}




\section{Introduction}

Multiple sclerosis (MS) is a chronic complex T cell-mediated autoimmune disease of the central nervous system (CNS). It is characterized by demyelination, axonal degeneration, and neuronal inflammation with a prominent neurodegenerative component [1]. In MS, several adhesion molecules are involved within the CNS in inflammatory and neurodegenerative processes [2]. It has been suggested that depletion of neural cell adhesion molecule (NCAM) is one of the factors associated with or possibly responsible for MS progression [3]. The NCAM, a member of the immunoglobulin superfamily, is expressed on both neurons and glial cells that has been intimately linked to axonal outgrowth, remyelination processes, guidance, and fasciculation and participate in the reparative mechanisms [4]. Isoforms of the NCAM carrying the linear homopolymer of alpha 2, 8-linked polysialic acid (PSA) also can provide unique properties in neural migration $[4,5]$. Therefore, NCAM-PSA appears to play a critical role in mediating precursor cell migration in the brain [6]. PSA is a glycan modification of the NCAM produced by the polysialyl transferases ST8SIA2 and ST8SIA4. PSA has been detected in MS plaques, but its adverse or beneficial role in remyelination is controversial. It has been recently shown that NCAM and ST8SIA2 promote oligodendrocyte differentiation and myelin repair, while ST8SIA4 delays oligodendrocyte differentiation, explaining its adverse role in remyelination [7].

It has also been suggested that not only regular exercise may benefit cognitive function by rescuing some hippocampal cellular and molecular impairments but also it has a direct neuroprotective effect in EAE, an animal model of MS. Several molecules such as NCAM and PAX7 are commonly used for detecting myogenic precursor cells and identifying their activation status. Caldow et al. [8] have demonstrated that the expression of the NCAM increase following acute resistance exercise to indicate a rapid initiation of the myofiber repair process. NCAM is expressed during myoblast proliferation and maturation [9], while PAX7 is expressed during quiescence and activation of myogenic precursor cells [10]. The NCAM and its PSA moiety are also essential for proper motor axon guidance in the developing neuromuscular system [11]. In MS patients, there is impaired balance and gait due to the process of demyelination as well as identified certain symptoms of motor disorders. These disorders can be estimated by quantifying neuromuscular and cortical parameters [12]. Here, we investigated the effect of forced and voluntary training pre- and postinduction of EAE on accumulation of NCA and PSA in neuromuscular junction denervation in plantaris and soleus muscles in $\mathrm{C} 57 \mathrm{BL} / 6$ female mice.

\section{Materials and Methods}

A total of 40 female C57BL/6 mice, 10-week-old, were randomly divided into four groups, including induced control groups without EAE induction, induced EAE without training, and forced and voluntary training groups. All animals have started training 4 weeks before experimental autoimmune encephalitis- (EAE-) induction. The mice were intraperitoneally anesthetized with an injection of xylazine $(5 \mathrm{mg} / \mathrm{kg})$ and ketamine $(50 \mathrm{mg} / \mathrm{kg})$. Myelin oligodendrocyte glycoprotein peptide 35-55 (300 $\mu \mathrm{g}$ in saline; MOG 35-55; KJ Ross-Petersen ApS, Denmark) was emulsified in an equal volume of complete Freund's adjuvant (CFA; Sigma, USA) containing $500 \mu \mathrm{g}$ of heatkilled Mycobacterium tuberculosis. This emulsion was then injected subcutaneously at the base of the tail of each mouse. The mice also received intraperitoneal injection of pertussis toxin (300 ng in $10 \mu \mathrm{l}$ PBS; List Biological Lab, USA) at the time of immunization and the same was repeated 48 hours later. To confirm EAE induction, animal clinical assessment of EAE was performed daily using a 15-point scoring system following immunization as reported previously by Giuliani et al. [13].

2.1. Compulsory Activity. Training groups performed the swimming program for $30 \mathrm{~min} /$ day, 5 times/week, for 4 weeks.

Mice were monitored during exercise sessions and encouraged to continue using a sponge. The exercise protocol was the same pre- and posttraining.

2.2. Voluntary Activity. Mice had access to a treadmill for one hour per day, 5 times/week, for 4 weeks in individual cage. The mileage was recorded using a computer. The mice were euthanized, and the plantaris and soleus muscles were then isolated for investigation of proteins expression using IHC.

2.3. IHC Staining. Localization of NCAM and PSA in plantaris and soleus muscles was studied using IHC. Tissue samples were fixed in $10 \%$ natural buffered formalin embedded in paraffin. Paraffin sample sections with a thickness of $10 \mu \mathrm{m}$ were used for IHC analysis as previously reported by Ghavideldarestani et al. [14]. In brief, the tissue slides were deparaffinized on an incubator for 1 hour and washed twice with xylene, each time for 10 minutes. The sections were incubated with blocking solution containing $4 \%$ goat serum for $30 \mathrm{~min}$ in PBS to prevent nonspecific staining by placing the slides in a humidifier chamber. The sections were then treated with 5\% normal donkey serum (NDS; Jackson Laboratories) diluted in PBS with $0.2 \%$ Triton-X100 (Sigma) for $1 \mathrm{~h}$ and were incubated overnight with anti-PSA, NCAM, and PSA-NCAM mouse monoclonal antibody diluted (Abcam, USA) at 1:500 in 0.1 M PBS containing 1\% (w/v) bovine serum albumin (BSA) and $1 \%(\mathrm{v} / \mathrm{v})$ normal goat serum in a dark sealed container at room temperature. The sections finally were exposed to fluorescein isothiocyanate(FITC-) conjugated goat anti-mouse IgM (Abcam, UK) diluted 1:200 for $3 \mathrm{~h}$. All sections were costained with $\alpha$-bungarotoxin (Abcam, USA) (1:100, Sigma-Aldrich, USA) to identify the neuromuscular junction. The slides were mounted with $4^{\prime}$, 6-diamidino-2-phenylindole (DAPI) (Vectashield mountingmedium with DAPI, Vector Laboratories, California, USA). 
2.4. Quantitative Evaluation of PSA-NCAM Expression. Images of the stained sections were taken using a fluorescent microscope. Quantitative image analysis was performed using ImageJ software package.

2.5. Statistical Analysis. The data were analyzed by SPSS version 22. Differences between the mean expressions of NCAM and PSA in various groups were analyzed by oneway ANOVA. Effects were considered significant at $p<0.05$. Data are presented as the mean \pm SD.

\section{Results}

The obtained data from the mean percentage expression of PSA in pre- and post-soleus muscle showed that the control group (58.71 $\pm 3.20 \%, 60.97 \pm 5.10 \%$, respectively) showed significantly higher mean percentage expression of PSA compared to SE $(21.73 \pm 2.24 \%, P<0.0001 ; 25.50 \pm 3.34 \%$, $P<0.0001$, respectively), ER $(45.41 \pm 3.81 \%, \quad P<0.0001$; $44.46 \pm 3.22 \%, P<0.0001$, respectively), and ES (35.40 \pm $3.73 \%, P<0.0001,38.50 \pm 4.49 \% ; P<0.0001$, respectively) groups (Figures 1 and 2). The mean percentage expression of PSA in pre- and post-soleus was significantly downregulated in the SE group compared with that of ER $(P<0.0001)$ and ES $(P<0.0001)$ groups (Figure 1$)$. Unlike pre-soleus muscle, there was significant difference in the mean percentage expression of PSA of post-soleus muscle in the ER group compared with that in the ES group (Figures 1 and 2).

The mean percentage expression of NCAM in pre- and post-soleus muscle showed that the control group $(61.36 \pm 2.56 \%, 64.96 \pm 2.03 \%$, respectively) expressed significantly higher percentage expression of NCAM than that in SE $(22.46 \pm 1.71 \%, P<0.0001 ; 25.87 \pm 3.69 \%, P<0.0001$, respectively), ER (51.92 $\pm 1.92 \%, P<0.0001 ; 49.41 \pm 2.81 \%$, $P<0.0001$, respectively), and ES $(39.13 \pm 2.48 \%, P<0.0001$; $46.07 \pm 2.91 \%, P<0.0001$, respectively).

The mean percentage expression of NCAM in pre- and post-soleus was significantly downregulated in the SE group compared with that in ER $(P<0.0001)$ and ES $(P<0.0001)$ groups (Figure 2$)$. However, there was no significant difference in the mean percentage expression of NCAM of pre- and post-soleus muscle in the SE group compared with that in ER and ES groups (Figures 3 and 4).

In pre- and post-EDL, the mean percentage of NCAM expression in the control group showed significant increase in the control group $(61.24 \pm 0.78,63.82 \pm 3.71 \%$, respectively) compared with that in SE $(24.43 \pm 4.75, P<0.0001$; $27.66 \pm 4.22 \%, P<0.0001 \%$, respectively), ER (51 \pm 8.43 , $P<0.0001 ; 48.61 \pm 1.59 \%, P<0.0001 \%$, respectively), and ES (39.23 $\pm 2.90, P<0.0001 ; 40.47 \pm 4.45 \%, P<0.0001)$ groups (Figure 3$)$. The mean percentage expression of NCAM in pre- and post-EDL was significantly downregulated in the SE group compared with that in ER $(P<0.0001)$ and ES $(P<0.0001)$ groups. There was no significant difference in the mean percentage expression of NCAM of pre- and postEDL in the SE group compared with that in ER and ES groups (Figures 5 and 6).
In pre- and post-EDL, the mean percentage of PSA expression in the control group showed significant increase in the control group $(52.06 \pm 1.78,56.27 \pm 3.81 \%$, respectively) compared with that in SE $(24.63 \pm 2.53, P<0.0001$; $24.41 \pm 1.75 \%, P<0.0001 \%$, respectively), ER (42.62 \pm 4.29 , $P<0.0001 ; 44.63 \pm 4.06 \%, P<0.0001 \%$, respectively), and ES $(41 \pm 2.16, \quad P<0.0001 ; 39.07 \pm 2.19 \%, \quad P<0.0001)$ groups (Figure 3 ). The mean percentage expression of PSA in preand post-EDL was significantly downregulated in the SE group compared with that in ER $(P<0.0001)$ and ES $(P<0.0001)$ groups. There was no significant difference in the mean percentage expression of NCAM of pre- and postEDL in the SE group compared with that in ER and ES groups (Figures 7 and 8).

\section{Discussion}

The aim of this study was to investigate the effect of forced and voluntary training pre- and postinduction of EAE on accumulation of NCAM and PSA in neuromuscular junction denervation in plantaris and soleus muscles in C57BL/6 female mice. A growing body of evidence has implicated NCAM and PSA as risk factors for major neuropsychiatric and neurodegenerative disorders [15]. The obtained data from the mean percentage expression of PSA and NCAM in pre- and post-soleus and plantaris muscles showed that the highest and lowest expression levels of PSA and NCAM belonged to the control and SE groups, respectively. The low expression levels of PSA and NCAM were detected in rat with MS without intervention. The PSA expression results suggest that compulsory and voluntary exercise can increase PSA and NCAM protein expression of Spre- and post-soleus and plantaris muscles in EAE animals. Moreover, voluntary wheel running exercise significantly increased PSA and NCAM protein expression compared with compulsory exercise in post-soleus muscle. However, the benefits of exercise in the expression of PSA and NCAM proteins in preand post-soleus and plantaris muscles in EAE animals are demonstrated in this study. Adhesion molecules such as PSA and NCAM are suggested to contribute in the different processes that result in the progress of lesions and neurodegeneration in MS $[2,7]$. PSA is a glycan modification of the NCAM that has been detected in MS plaques, but its beneficial or adverse role in remyelination is controversial [7].

It has been suggested that PSA-NCAM may modulate the functional interaction between neurotrophin brain-derived neurotrophic factor (BDNF) and its high and low affinity receptors. So, the distribution of the BDNF and PSANCAM modulates BDNF signaling [16]. It has been recently shown that the forced high intensity exercise may diminish the protein concentration of BDNF in soleus muscle [17].

The endoplasmic reticulum (ER) modulates various physiological processes including protein folding and calcium homeostasis to maintain cellular homeostasis [18]. Multiple pathologic factors, such as oxidative stress triggering prolonged disorders or distress on ER homeostasis, resulting in the unfolded protein response pathway in the ER lumen is known as ER stress [19]. Changes in the 

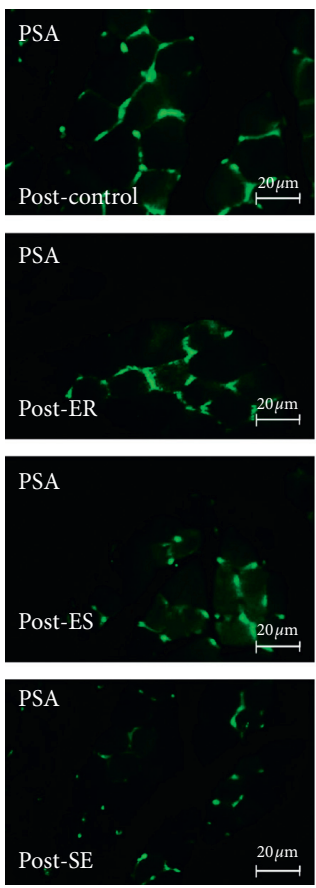
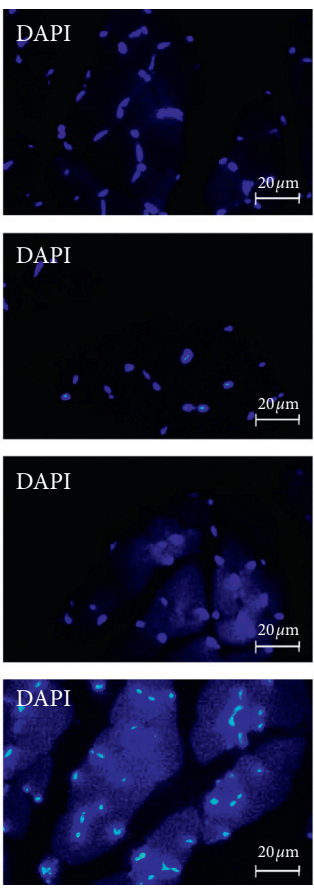

(a)
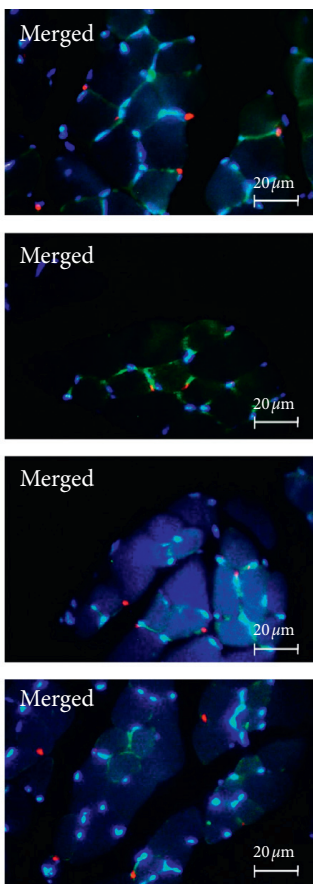

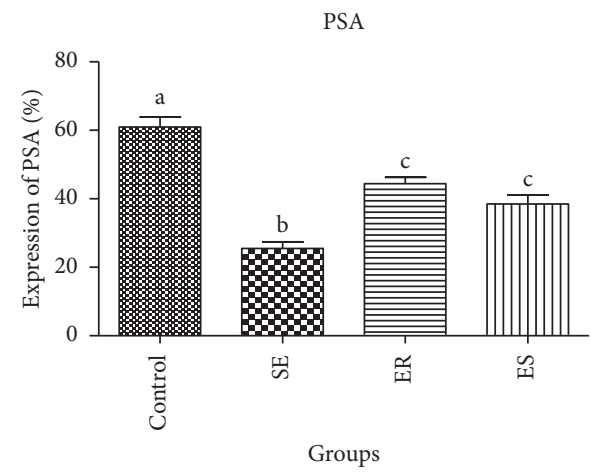

(b)

Figure 1: The expression of PSA post-soleus muscle. The mean percentage of PSA expression in control group was significantly higher than those of compared to SE $(P<0.0001)$, ER $(P<0.0001)$, ES $(P<0.0001)$ groups. The mean percentage expression of PSA in post-soleus was significantly down-regulated in SE group compared to ER $(P<0.0001)$ and ES $(P<0.0001)$ groups. There was significant difference in the mean percentage expression of PSA of post-soleus muscle in ER group compared to ES group.
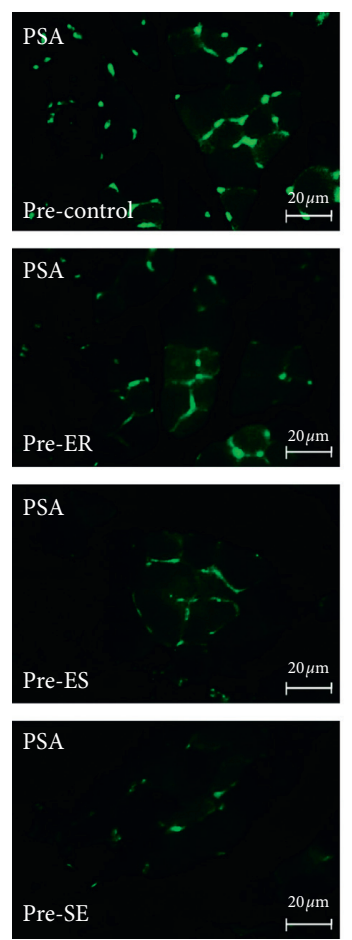
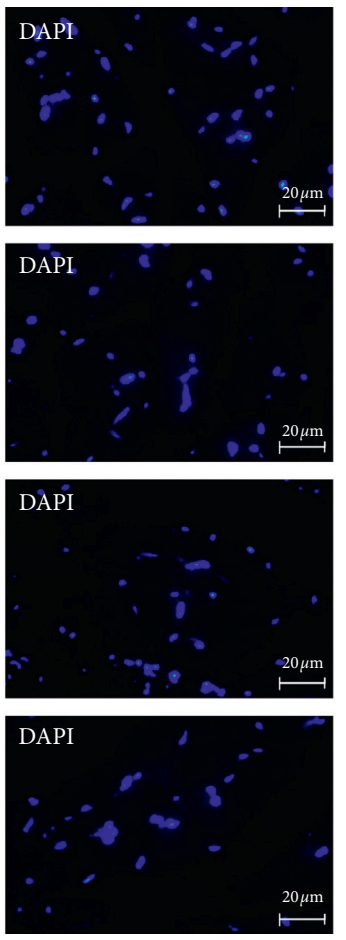

(a)
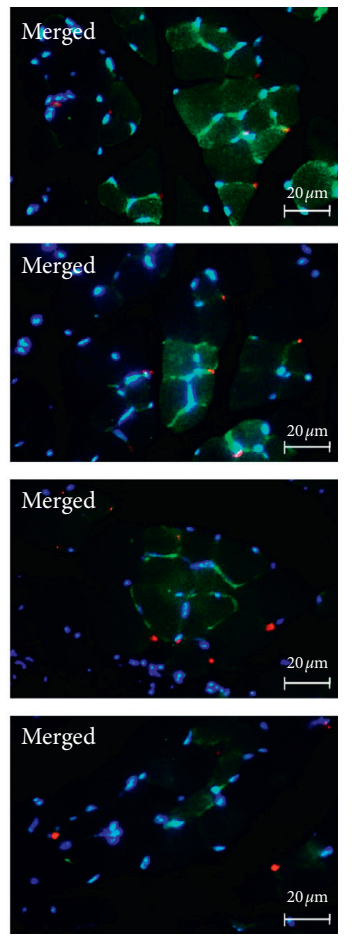

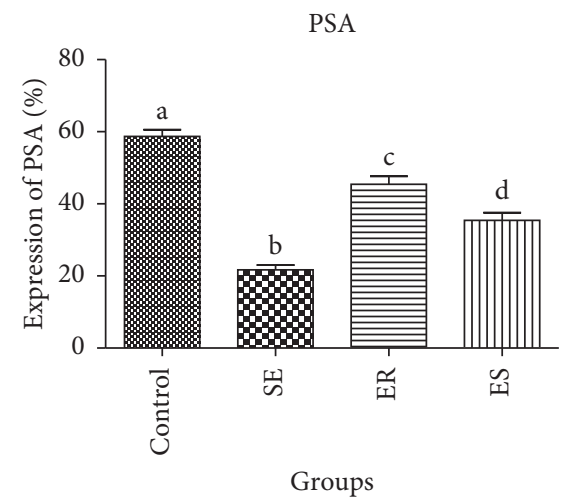

(b)

FIGURE 2: The expression of PSA pre-soleus muscle. The mean percentage of PSA expression in control group was significantly higher than those of compared to SE $(P<0.0001)$, ER $(P<0.0001)$, ES $(P<0.0001)$ groups. The mean percentage expression of PSA in post-soleus was significantly down-regulated in SE group compared to ER $(P<0.0001)$ and ES $(P<0.0001)$ groups. 

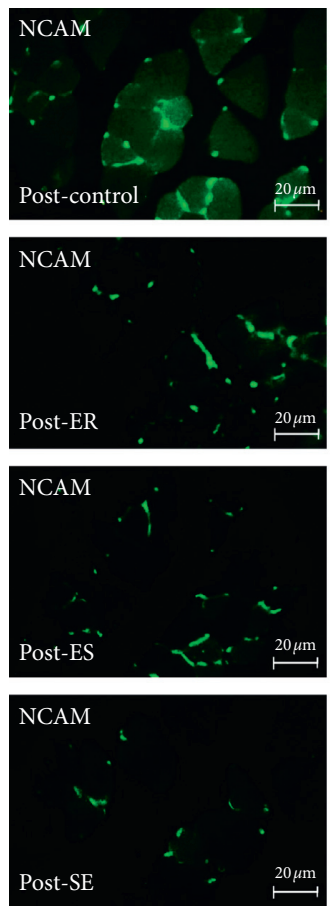
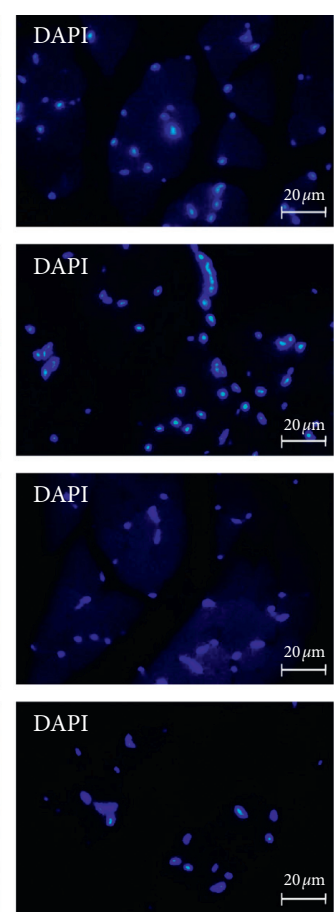

(a)
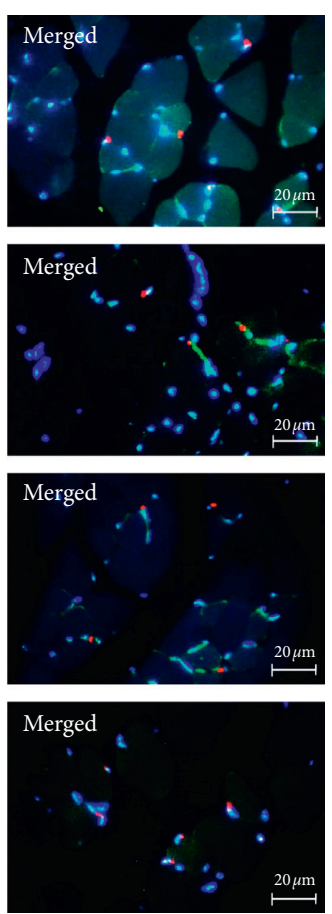

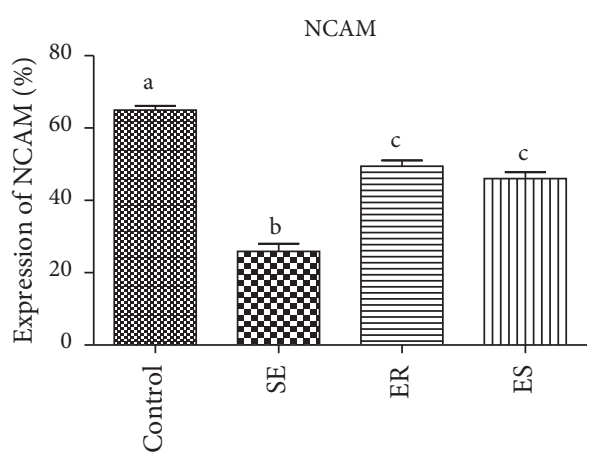

Groups

(b)

Figure 3: The expression of NCAM in post-soleus muscle. The mean percentage of NCAM expression in control group was significantly higher than those of compared to SE $(P<0.0001)$, ER $(P<0.0001)$, ES $(P<0.0001)$ groups. The mean percentage expression of NCAM in post-soleus was significantly down-regulated in SE group compared to ER $(P<0.0001)$ and ES $(P<0.0001)$ groups.
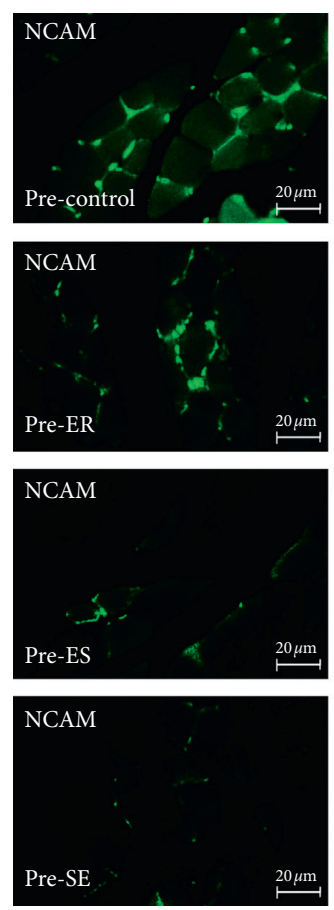
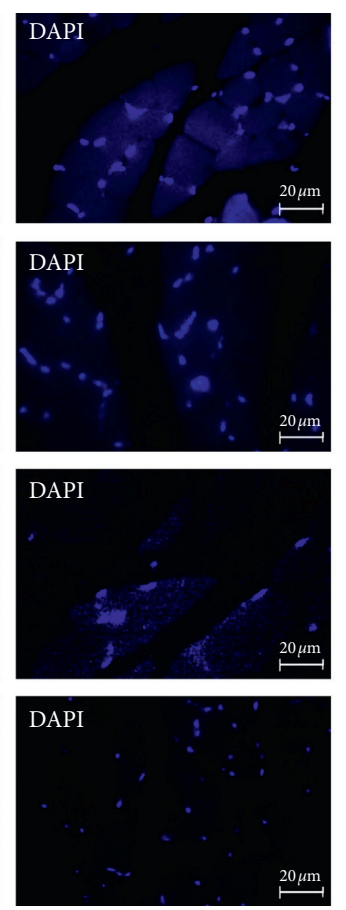

(a)
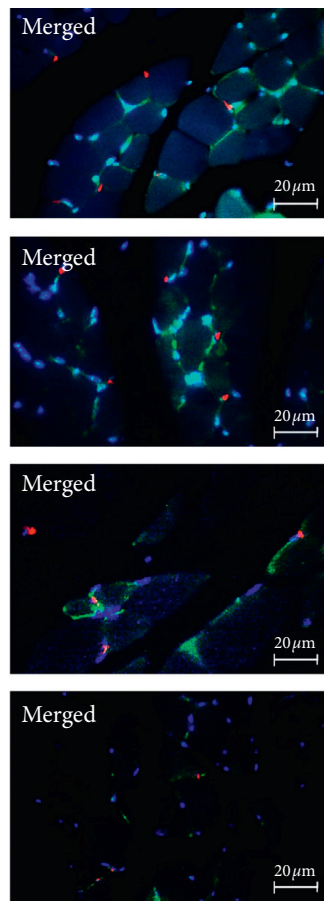

Groups

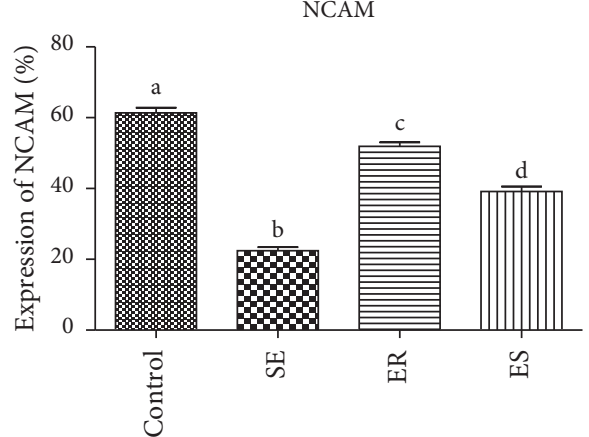

Figure 4: The expression of NCAM in pre-soleus muscle. The mean percentage of NCAM expression in control group was significantly higher than those of compared to SE $(P<0.0001)$, ER $(P<0.0001)$, ES $(P<0.0001)$ groups. The mean percentage expression of NCAM in pre-soleous was significantly down-regulated in SE group compared to ER $(P<0.0001)$ and ES $(P<0.0001)$ groups. 

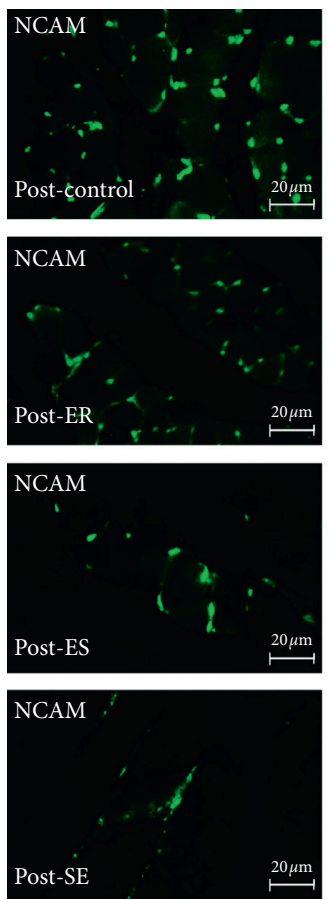
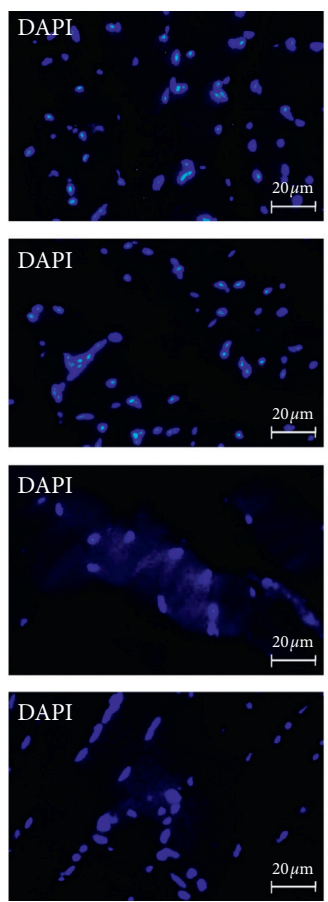

(a)
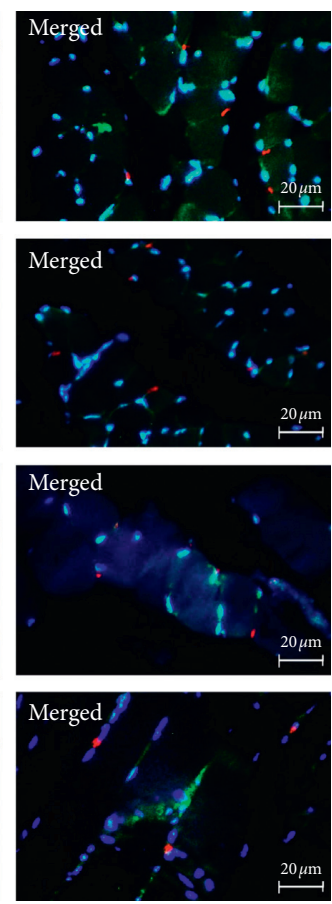

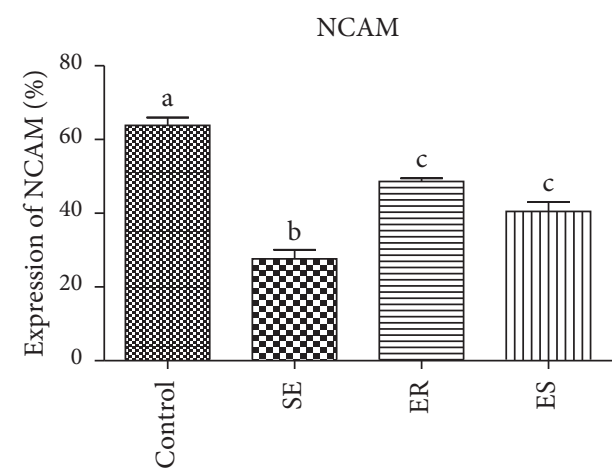

Groups

(b)

FIGURE 5: In post-EDL, the mean percentage of NCAM expression in control group showed significant increase in control group compared to SE $(P<0.0001)$, ER $(P<0.0001)$, ES $(P<0.0001)$ groups. The mean percentage expression of NCAM in post-EDL was significantly downregulated in SE group compared to ER $(P<0.0001)$ and ES $(P<0.0001)$ groups.
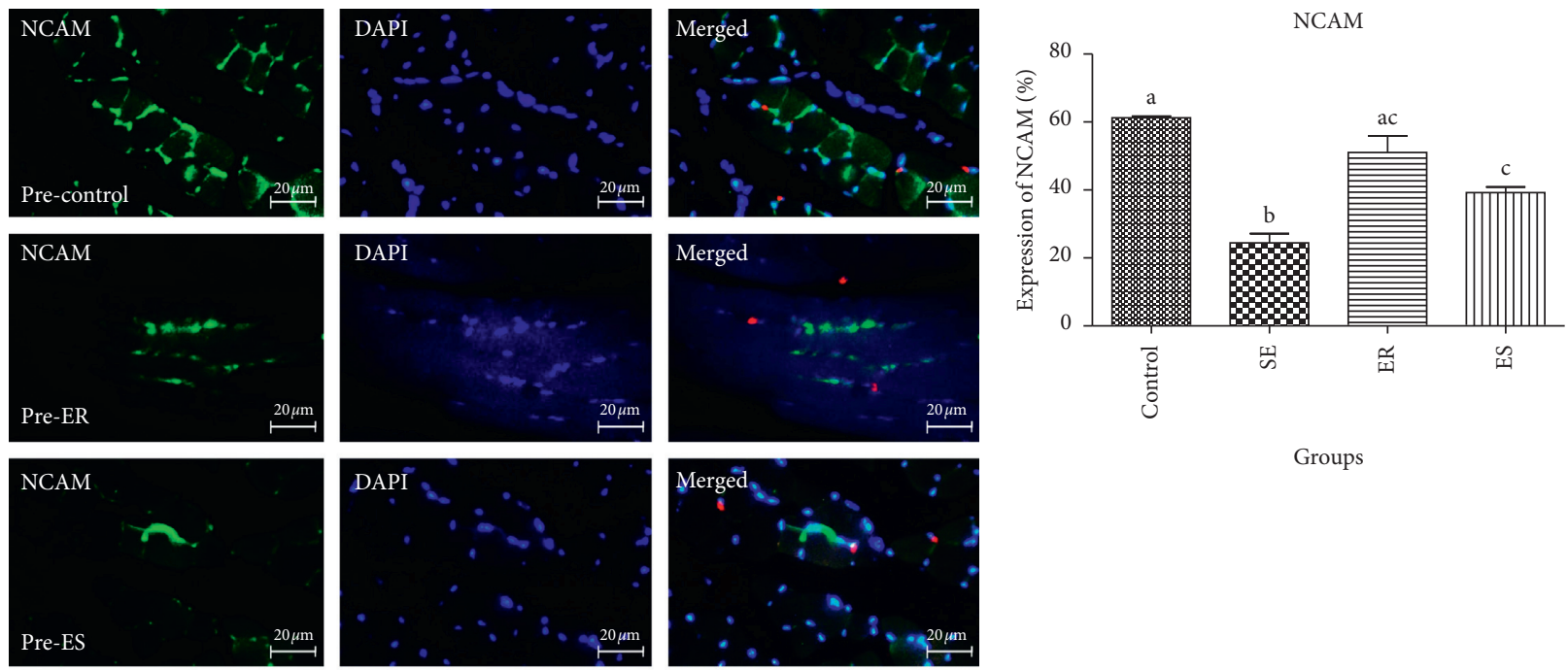

Groups
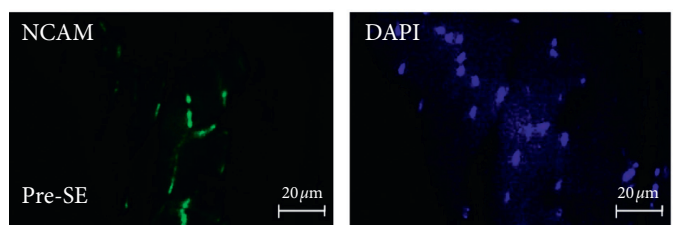

(a)

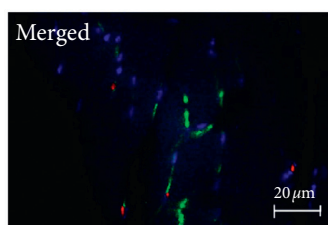

$20 \mu \mathrm{m}$

(b)

FIGURE 6: In pre-EDL, the mean percentage of NCAM expression in control group showed significant increase in control group compared to SE $(P<0.0001)$, ER $(P<0.0001)$, ES $(P<0.0001)$ groups. The mean percentage expression of NCAM in post-EDL was significantly downregulated in SE group compared to ER $(P<0.0001)$ and ES $(P<0.0001)$ groups. 

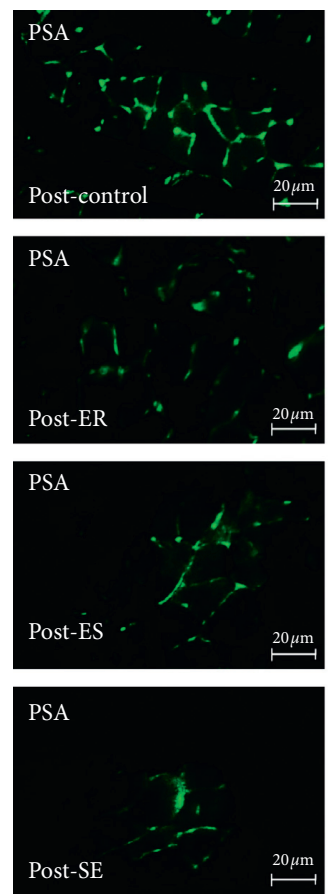
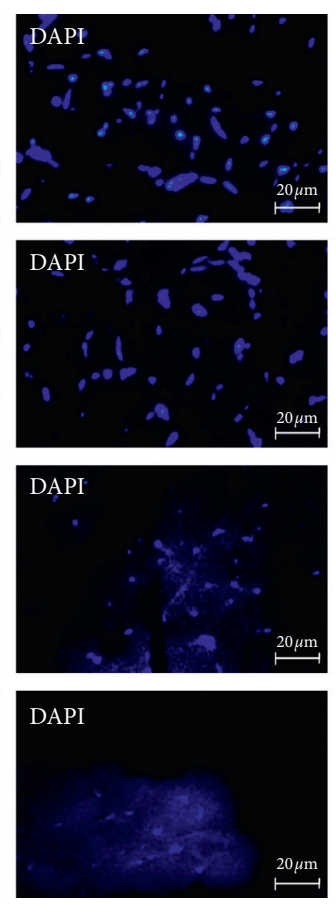

(a)
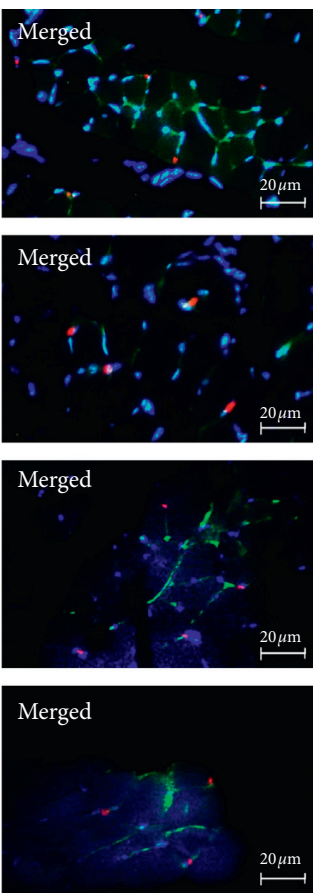

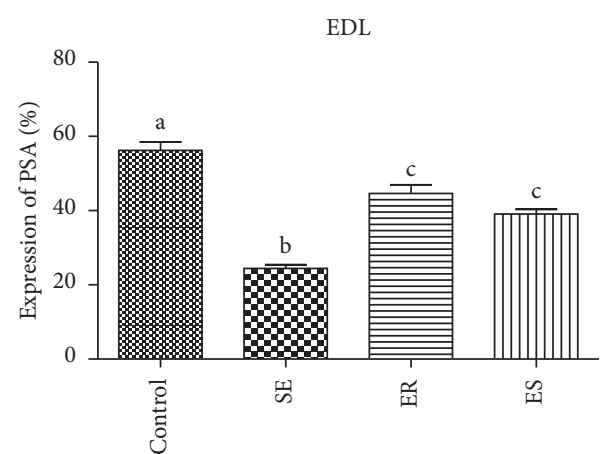

Groups

(b)

FIGURE 7: In post-EDL, the mean percentage of PSA expression in control group showed significant increase in control group compared to SE $(P<0.0001)$, ER $(P<0.0001)$, ES $(41 \pm 2.16, P<0.0001)$ groups. The mean percentage expression of PSA in post-EDL was significantly down-regulated in SE group compared to ER $(P<0.0001)$ and ES $(P<0.0001)$ groups.
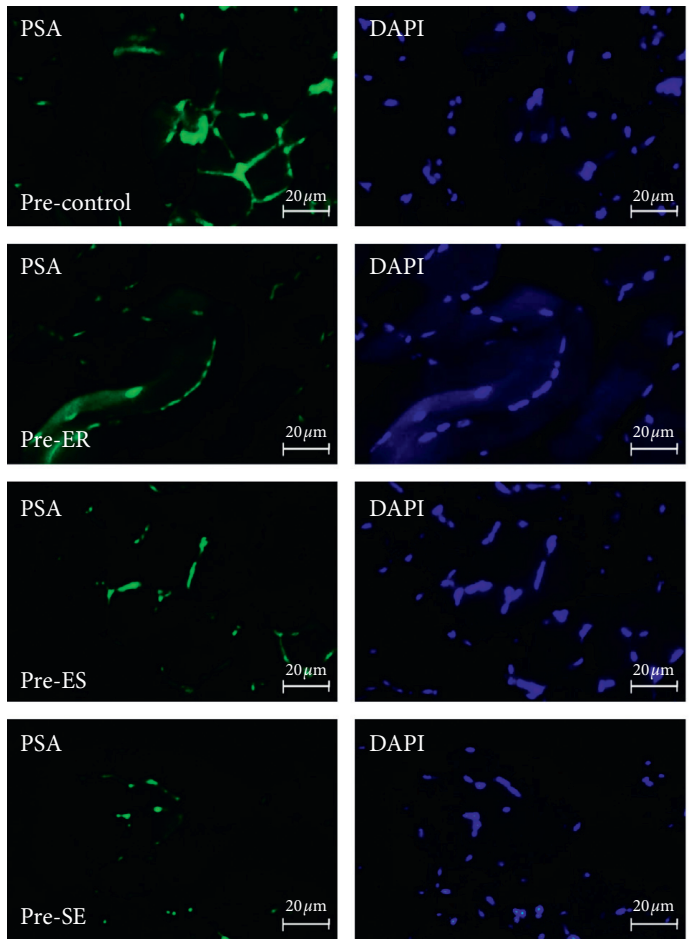

(a)
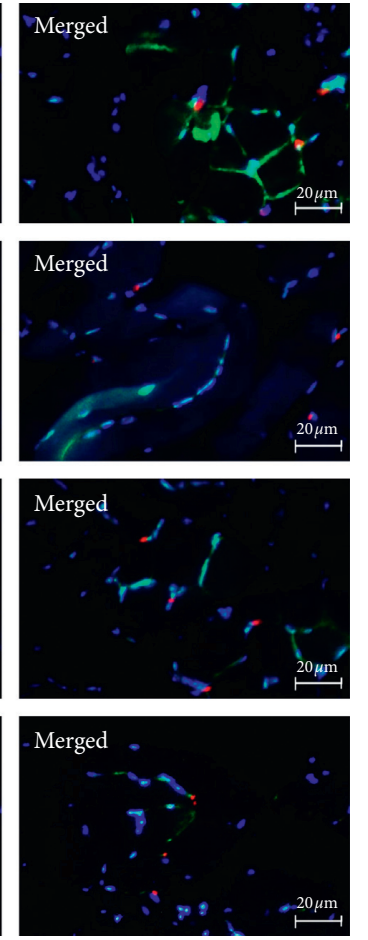

PSA

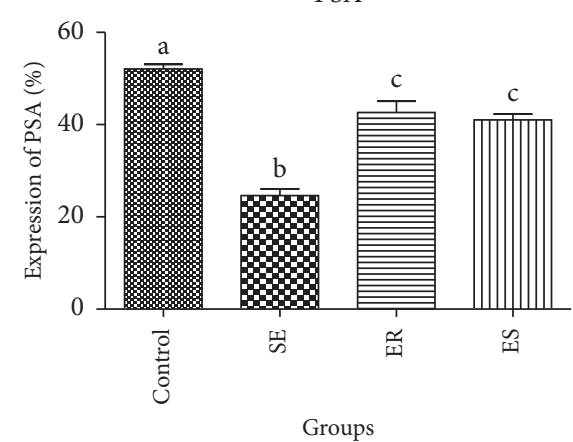

(b)

FIGURE 8: In pre-EDL, the mean percentage of PSA expression in control group showed significant increase in control group compared to SE $(P<0.0001)$, ER $(P<0.0001)$, ES $(41 \pm 2.16, P<0.0001)$ groups. The mean percentage expression of PSA in pre-EDL was significantly downregulated in SE group compared to ER $(P<0.0001)$ and ES $(P<0.0001)$ groups. 
inflammatory profile and inhibition of immune responses induced by exercise cause alterations in the oxidative stress parameters [20]. Molecular adhesion molecules such as NCAM protein is synthesized in the ER as a membranebound glycoprotein that can undergo various posttranslational modifications before reaching the cell surface. Collectively, the ameliorative effect of exercise on ER stressassociated risk factors such as inflammation and oxidative stress can be important in the expression of molecular adhesion [21]. Therefore, the low expression of NCAM and PSA in EAE mice may attribute to the effect of inflammation and stress oxidative activity in neuromuscular junction as well as axonal damage. In contrast, the higher expression level of these proteins in voluntary and compulsory activity mice groups may attribute to the ameliorative effect of exercise [19]. The reductions in autoimmune cell infiltration, delay in onset of clinical disability, attenuation of severity, and preservation of axons and motor neurons in the lumbar spinal cord of mice with EAE with voluntary wheel running have been previously reported [22]. Exercise has also been associated with preserving or enhancing of physical function in human with MS, indicating the neuroprotection role of exercise [23]. It has been shown that the proteins deterioration occurs directly or indirectly following oxidative stress including damage to specific amino acid residues, peroxidation, changes, or degradation in their tertiary structure and lose of enzymatic activity [24]. These changes lead to alterations in the type and level of cellular proteins especially NCAM as well as physiological cellular functions [24]. The association of immunomodulatory effects of exercise with the expression of tight-junction proteins and reduced adhesion molecules expression, especially of extracellular vascular cell adhesion molecule 1 (VCAM-1), platelet and endothelial cell adhesion molecule 1 (PECAM-1), and intercellular adhesion molecules 1 (ICAM-1) have been previously reported $[25,26]$. It has been recently shown that the biological pathways linking these adhesion molecules alter in MS [2]. Cell adhesion molecules such as NCAM play an important role in signal transduction cell, migration and proliferation, neurite outgrowth and fasciculation, and synaptogenesis and synaptic plasticity [27]. NCAM promotes and regulates synaptic stability and strongly influences neurotransmission. NCAM functions depend on its glycosylation, particularly on polysialylation, i.e., attachment of PSA [28]. The role of NCAM and its associated PSA as an important mediator of synaptic plasticity has been widely recognized [28]. It has been demonstrated that PSA gene and protein expression level are regulated in an age-, cell type-, and activity-dependent manner [15]. Spasticity, perceived by patients as muscle rigidity and spasms, is a common symptom in MS [29] that may be reduced by exercise.

In conclusion, the relationship between increasing levels of NCAM and PSA protein expression and voluntary and compulsory activities was detectable both in pre- and postsoleus and plantaris. However, voluntary activity resulted in more expression levels of NCAM and PSA than those of the compulsory one. In conclusion, since it has been suggested that depletion of NCAM is one of the factors associated with or possibly responsible for MS progression, these findings show exercise MS progression may be reduced by increasing expression of exercise-related adhesion molecule such as NCAM and PSA (a glycan modification of the NCAM).

\section{Data Availability}

All data used to support the findings of this study can be made available from the corresponding author upon request (mrkordi@ut.ac.ir).

\section{Conflicts of Interest}

The authors declare that they have no conflicts of interest.

\section{References}

[1] C. Fenoglio, E. Oldoni, M. Serpente et al., "LncRNAs expression profile in peripheral blood mononuclear cells from multiple sclerosis patients," Journal of Neuroimmunology, vol. 324, pp. 129-135, 2018.

[2] N. Ziliotto, R. Zivadinov, D. Jakimovski et al., "Plasma levels of soluble NCAM in multiple sclerosis," Journal of the Neurological Sciences, vol. 396, pp. 36-41, 2019.

[3] S. Gnanapavan, P. Ho, W. Heywood et al., "Progression in multiple sclerosis is associated with low endogenous NCAM," Journal of Neurochemistry, vol. 125, no. 5, pp. 766-773, 2013.

[4] U. Rutishauser, "Polysialic acid in the plasticity of the developing and adult vertebrate nervous system," Nature Reviews Neuroscience, vol. 9, no. 1, pp. 26-35, 2008.

[5] E. Gascon, L. Vutskits, and J. Z. Kiss, "Polysialic acid-neural cell adhesion molecule in brain plasticity: from synapses to integration of new neurons," Brain Research Reviews, vol. 56, no. 1, pp. 101-118, 2007.

[6] T. Glaser, C. Brose, I. Franceschini et al., "Neural cell adhesion molecule polysialylation enhances the sensitivity of embryonic stem cell-derived neural precursors to migration guidance cues," Stem Cells, vol. 25, no. 12, pp. 3016-3025, 2007.

[7] S. Werneburg, H. L. S. Fuchs, I. Albers et al., "Polysialylation at early stages of oligodendrocyte differentiation promotes myelin repair," The Journal of Neuroscience, vol. 37, no. 34, pp. 8131-8141, 2017.

[8] M. K. Caldow, E. E. Thomas, M. J. Dale, G. R. Tomkinson, J. D. Buckley, and D. Cameron-Smith, "Early myogenic responses to acute exercise before and after resistance training in young men," Physiological Reports, vol. 3, no. 9, 2015.

[9] M. Ishido, M. Uda, M. Masuhara, and K. Kami, "Alterations of M-cadherin, neural cell adhesion molecule and beta-catenin expression in satellite cells during overload-induced skeletal muscle hypertrophy," Acta Physiologica, vol. 187, no. 3, pp. 407-418, 2006.

[10] R. W. Ten Broek, S. Grefte, and J. W. Von den Hoff, "Regulatory factors and cell populations involved in skeletal muscle regeneration," Journal of Cellular Physiology, vol. 224, no. 224, pp. 7-16, 2010.

[11] C. K. Franz, U. Rutishauser, and V. F. Rafuse, "Polysialylated neural cell adhesion molecule is necessary for selective targeting of regenerating motor neurons," Journal of Neuroscience, vol. 25, no. 8, pp. 2081-2091, 2005.

[12] M. C. Neamţu, L. Rusu, O. M Neamţu et al., "Analysis of neuromuscular parameters in patients with multiple sclerosis and gait disorders," Romanian Journal of Morphology and Embryology, vol. 55, no. 4, pp. 1423-1428, 2014. 
[13] F. Giuliani, W. Hader, and V. W. Yong, "Minocycline attenuates $\mathrm{T}$ cell and microglia activity to impair cytokine production in T cell-microglia interaction," Journal of Leukocyte Biology, vol. 78, no. 1, pp. 135-143, 2005.

[14] M. Ghavideldarestani, A. E. Butler, S. Shirian, and S. L. Atkin, "Expression and localization of transient receptor potential channels in the bovine uterus epithelium throughout the estrous cycle," Molecular Biology Reports, vol. 46, no. 4, pp. 4077-4084, 2019.

[15] L. H. Brennaman and P. F. Maness, "NCAM in neuropsychiatric and neurodegenerative disorders," Advances in Experimental Medicine and Biology, vol. 663, pp. 299-317, 2010.

[16] M. Quartu, M. P. Serra, M. Boi, T. Melis, R. Ambu, and M. Del Fiacco, "Brain-derived neurotrophic factor (BDNF) and polysialylated-neural cell adhesion molecule (PSA-NCAM): codistribution in the human brainstem precerebellar nuclei from prenatal to adult age," Brain Research, vol. 1363, pp. 49-62, 2010.

[17] A. Jiménez-Maldonado, J. Cerna-Cortés, E. M. CastroRodríguez et al., "Effects of moderate- and high-intensity chronic exercise on brain-derived neurotrophic factor expression in fast and slow muscles," Muscle \& Nerve, vol. 53, no. 3, pp. 446-451, 2016.

[18] S. Shinozaki, "A deficiency of herp, an endoplasmic reticulum stress protein, suppresses atherosclerosis in ApoE knockout mice by attenuating inflammatory responses," PLoS One, vol. 8, Article ID e75249, 2013.

[19] J. Hong, K. Kim, E Park et al., "Exercise ameliorates endoplasmic reticulum stress-mediated vascular dysfunction in mesenteric arteries in atherosclerosis," Scientific Reports, vol. 8, no. 1, p. 7938, 2018.

[20] P. S. Souza, E. D. Gonçalves, G. S. Pedroso et al., "Physical exercise attenuates experimental autoimmune encephalomyelitis by inhibiting peripheral immune response and bloodbrain barrier disruption," Molecular Neurobiology, vol. 54, no. 6, pp. 4723-4737, 2017.

[21] N. Bakunina, C. M. Pariante, and P. A. Zunszain, "Immune mechanisms linked to depression via oxidative stress and neuroprogression," Immunology, vol. 144, no. 3, pp. 365-373, 2015.

[22] W. M. Pryor, K. G. Freeman, R. D. Larson, G. L. Edwards, and L. J. White, "Chronic exercise confers neuroprotection in experimental autoimmune encephalomyelitis," Journal of Neuroscience Research, vol. 93, no. 5, pp. 697-706, 2015.

[23] V. Castellano and L. J. White, "Serum brain-derived neurotrophic factor response to aerobic exercise in multiple sclerosis," Journal of the Neurological Sciences, vol. 269, no. 1-2, pp. 85-91, 2008.

[24] R. Kohen and A. Nyska, "Invited review: oxidation of biological systems: oxidative stress phenomena, antioxidants, redox reactions, and methods for their quantification," Toxicologic Pathology, vol. 30, no. 6, pp. 620-650, 2002.

[25] J. I. Alvarez, R. Cayrol, and A. Prat, "Disruption of central nervous system barriers in multiple sclerosis," Biochimica et Biophysica Acta (BBA)-Molecular Basis of Disease, vol. 1812, no. 2, pp. 252-264, 2011.

[26] C. Larochelle, J. I. Alvarez, and A. Prat, "How do immune cells overcome the blood-brain barrier in multiple sclerosis?" FEBS Letters, vol. 585, no. 23, pp. 3770-3780, 2011.

[27] P. F. Maness and M. Schachner, "Neural recognition molecules of the immunoglobulin superfamily: signaling transducers of axon guidance and neuronal migration," Nature Neuroscience, vol. 10, no. 1, pp. 19-26, 2007.
[28] G. Kochlamazashvili, O. Senkov, S. Grebenyuk et al., "Neural cell adhesion molecule-associated polysialic acid regulates synaptic plasticity and learning by restraining the signaling through GluN2B-containing NMDA receptors," Journal of Neuroscience, vol. 30, no. 11, pp. 4171-4183, 2010.

[29] G. Izquierdo, "Multiple sclerosis symptoms and spasticity management: new data," Neurodegenerative Disease Management, vol. 7, no. 6s, pp. 7-11, 2017. 